ISSN: 0213-2052 - eISSN: 2530-4100

DOI: https://doi.org/10.14201/shha202139309335

\title{
ESPECIALISTAS EN EL EJÉRCITO ROMANO. LA FUNCIÓN MILITAR DE LOS FRUMENTARII DURANTE EL PRINCIPADO
}

\section{Specialists in the Roman army: the military role of the Frumentarii during the Principate}

\author{
Miguel Pablo SANCHO GÓMEZ \\ Universidad Católica de Murcia \\ mpsancho@ucam.edu
}

Fecha de recepción: 25-06-2020 Fecha de aceptación: 28-05-2021

ORCID: https://orcid.org/0000-0003-0528-1721

RESUMEN: Este artículo ofrece una perspectiva poco explorada sobre las funciones y evolución de un cuerpo especial perteneciente al ejército romano, los frumentarii. Asociados a la categoría de principales, al abastecimiento y distribución de suministros, y especialmente de grano, como su nombre parece indicar, fueron prontamente encargados de una gran cantidad de actividades adicionales. Misiones para transportar materiales de lujo a Roma, «espionaje», vigilancia, servicio de policía, asesinatos, recogida de tributos y supervisión de la defensa en las provincias fueron algunos de los dispares, aunque esenciales, cometidos que la tropa llevó a cabo hasta finales del siglo III. Aunque al parecer su pésima reputación propició la disolución del cuerpo, durante muchos años fueron los ojos y oídos de los emperadores. Intentaremos explicar su desarrollo e importancia en la sociedad romana, haciendo hincapié en la pervivencia de sus habilidades militares. 
Palabras clave: frumentarii; exploradores; «espías»; asesinos; ejército romano; Imperio romano; Principado.

ABSTRACT: This article offers a fresh perspective about the role and evolution of one of the sections of the Roman army, the Frumentarii. Linked with the rank of principales, and with the supply and provisions, especially grain, as their name seems to indicate, this squad took at an early date a great deal of additional functions. Missions to drive and protect luxury stuff to Rome, "espionage", surveillance, police duties, murder, collecting taxes and provincial defense supervision, were some of the unalike but key functions carried by them until the end of Third Century. Although it seems as their infamous reputation fostered their disbandment, for long years they were the emperors' ears and eyes. We will try to explain their development and importance in the Roman society, highlighting the survival of their military skills.

Keywords: Frumentarii; scouts; "spies»; assassins; Roman army; Roman Empire; Principate.

\section{Definición DEL TÉRMino}

Las unidades de especialistas militares resultan una constante a lo largo de la historia. Roma, concretamente, siempre contó con soldados de los que se esperaba que se encargasen de una gran variedad de cometidos y misiones específicos. Este es el caso de los frumentarii, de los que ofreceremos un breve recorrido histórico desde los inicios altoimperiales hasta su desbandada al final del Principado. El tema presenta un gran interés, dados los numerosos interrogantes por descifrar en todo lo concerniente a estos individuos. Se debe diferenciar la existencia de ese cargo en el ejército de la atribución a ciertas tropas de funciones concretas, unida a la aparición de los principales y officiales en el ejército romano ${ }^{1}$.

Un frumentarius, aunque no fuese un miles legionis, en el sentido de «soldado raso", era miembro de una legión ${ }^{2}$. Su nombre, posiblemente asociado al trigo (frumentum), revela una vinculación, al menos inicial, con el aprovisionamiento ${ }^{3}$. Es también posible que tal término derive de la obtención de un reparto suplementario de grano, por llevar a cabo

1. Véase Speidel 2001, 50-61.

2. Véanse Fiebiger 1912, cols. 1690-224; Vaglieri 1922, 221; Krenkel 1990, 1009; Clauss 1973, 82-109; Dvornik 1974, 88-121 (especialmente 101-118), y Paschoud 1983, 215-244.

3. Sinnigen 1962, 211-224: "As their name implies, they were originally and probably throughout their history, connected with frumentum, grain, the staff of life». 
funciones diferenciadas del resto, circunstancia común entre los principales/officiales ${ }^{4}$. Los frumentarii fueron por tanto desgajados de su unidad de origen por ciertas capacidades concretas o un entrenamiento especial que los hacían aptos para misiones distintivas. Probablemente, desde inicios del siglo II, este cargo aparecía ya entre los mencionados principales. Eso implica que disfrutaban de una serie de beneficios — en lo referido a la paga, raciones y a la exención de ciertas tareas- debido a su especialización. Así, podían promocionar directamente a determinados cargos 5 . Aunque la investigación moderna no ha llegado a un consenso respecto a los orígenes del cuerpo, algunos autores afirman que no hay pruebas de peso para relacionarlos con los soldados de época republicana encargados del abastecimiento de grano; incluso parte de la historiografía especula que dicha tarea no se encontrase entre sus cometidos durante el Imperio, aunque tal cuestión dista de estar clara ${ }^{6}$. Lo cierto es que ya fueron mencionados diferencialmente por Julio César, que los asocia al suministro en sus Comentarios de las guerras de las Galias ${ }^{7}$.

F. Dvornik ha querido ver como frumentarii a los agentes enviados por Octavio a diversos lugares de Italia para contrarrestar la propaganda de su enemigo Marco Antonio, aunque esto es incierto ${ }^{8}$. Los primeros testimonios de un sistema central de información y mensajería imperial se sitúan durante el reinado de Augusto, donde vemos especialistas realizando labores de correos o emisarios entre las autoridades provinciales y el gobierno central en Roma, cubriendo también las funciones de enlace entre la unidad a la que pertenecían y el Prefecto del Pretorio9. La

4. Véanse Rankov 2007, 1171-1172; Fraipoint 2019, 18; Breeze 1971, 134. Su rango superior es el de beneficiarius consularis, al que pudo ascender con frecuencia (AE 1917 18, 57); Clauss 1973, 109. El frumentarius como principalis en Domaszewski-Dobson 1967, 35. Una ración extra de grano por funciones especiales de gran importancia aparece referida en Veg. Mil. I 13: Ita autem seuere apud maiores exercitii disciplina seruata est, ut et doctores armorum duplis remunerarentur annonis et milites, qui parum in illa prolusione profecerant, pro frumento bordeum cogerentur accipere, nec ante eis in tritico redderetur annona, quam sub praesentia praefecti legionis, tribunorum uel principiorum experimentis datis ostendissent se omnia, quae erant in militari arte, conplere.

5. Véanse Brunt 1950, 50-71; Speidel 1992, 87-106.

6. Rankov 2006, 135; Palao Vicente 2016, 151-152. Véase la n. 18.

7. Caes. BGall, VIII 35, 4: Quorum strepitum uigiles castrorum cum sensissent, exploratoresque missi quae gererentur renuntiassent, Caninius celeriter cum cohortibus armatis ex proximis castellis in frumentarios sub ipsam lucem impetum fecit; en particular nos parece interesante su función acompañando a los convoyes de cereales, parece que como guarnición o escolta. Véase también Sheldon 2006, 120-139.

8. Dvornik 1974, 102.

9. Suet. Aug. 49, 5: Et quo celerius ac sub manum adnuntiari cognoscique posset, quid in prouincia quaque gereretur, iuuenes primo modicis interuallis per militaris uias, 
ubicación del Castra Praetoria fuera del pomerium, entre las vías Nomentana y Salaria, facilitaría el empleo regular de soldados como mensajeros, haciendo más seguras las comunicaciones; así, los militares pasarían a acaparar las misiones de información ${ }^{10}$. Los continuos viajes y las estrechas relaciones de las tropas acuarteladas en Roma con las vías de comunicación y calzadas convirtieron posteriormente a los frumentarii en individuos que atesoraban conocimientos relevantes de sus destinos, guarniciones, etc. Esto resultaba especialmente cierto cuando se hallaban en las zonas fronterizas del Imperio, donde se estacionaban, como es sabido, un número significativo de legiones. No obstante, en mayor medida, los frumentarii se situaron en Roma, en las capitales provinciales y en otros núcleos urbanos importantes. La cercanía con la observación y la recogida de información a nivel local tuvo amplias consecuencias posteriores ${ }^{11}$.

El emperador Domiciano fue esencial en el desarrollo de un servicio de información militar, por lo que se le relaciona fuertemente con el rol definitivo del cuerpo $^{12}$. Los oficiales de inteligencia, ya desarrollados por Augusto, que había comprobado la utilidad de las buenas comunicaciones y el manejo rápido de las noticias, estaban en posición notable para obtener datos sobre la población local, el ejército, y al mismo tiempo mantener informados a los gobernadores, ya que transitaban continuamente los caminos debido a sus tareas. Podían, por tanto, enviar informes sobre cualquier cuestión de interés. Además, un correo o mensajero

dehinc uehicula disposuit. Commodius id uisum est, ut qui a loco idem perferunt litteras, interrogari guoque, si quid res exigant, possint. Augusto aseguró la eficacia de sus mensajeros proporcionándoles un medio de transporte oficial, el uehiculatio, más tarde conocido como el cursus publicus. Sistema de transporte estatal para cartas y órdenes oficiales, era utilizado para comunicación y misiones militares, así como algunas cuestiones fiscales. Véase Fraipoint 2019, 11-12, que alude a cursores o tabellari como mensajeros. Parece que fue Sejano, en tiempos de Tiberio, quien, al concentrar las nueve cohortes de pretorianos en un solo campamento, comenzó el proceso por el cual el Prefecto del Pretorio pasó a controlar casi todas las tropas de la ciudad, algo que afectaría también a los frumentarii. Así, Tac. Ann. 4, 2. Véase también Sheldon 2006, 143-163.

10. Cass. Dio 57 19, 6.

11. Véase Rankov 2002, 129-140. Véase también Perea Yébenes 1999, 235. Sabemos que acudían a los collegia de los speculatores, con los que existía cercanía profesional, o "Colegialidad". De tal modo, podemos ver a un frumentarius custodiando la restauración de la schola speculatorum en Aquincum (cerca de la actual Budapest): CIL III 3524. Un caso similar en CIL III $14479=$ AE 1901, $154=$ IDR III/5 426 (Apulum). Parece que se dio una conexión evidente entre frumentarii, stationes, beneficiarii y speculatores; véanse Palao Vicente 2016, 151, y Nelis-Clément 2000, 211-268.

12. Así, Crowdy 2006, 22. También Sinnigen 1961, 65: «I believe that there are no reasons to link the frumentarii with the creation of the imperial praetorium by Augustus or with the arrest and detention of St. Paul during Nero's reign». 
imperial siempre obtenía libre paso, lo que facilitaba grandemente sus misiones $^{13}$. Los frumentarii de este modo comenzarían a desarrollarse en plenitud durante el siglo I, sustituyendo progresivamente a los antiguos speculatores como mensajeros ${ }^{14}$. Resulta evidente que estos elementos se ganaron la confianza de los gobernantes, pues, según parece, fueron evolucionando a la categoría de "espías" eventuales, cometido que ya en parte realizaban en la sociedad romana algunos libertos y los delatores ${ }^{15}$.

Una segunda versión sobre el origen del cuerpo defiende que fueron creados por Trajano, alrededor del año $100^{16}$. Como individuo en contacto con el lugareño y buen conocedor de la sociedad local, un frumentarius estaba en condiciones inmejorables para recabar informaciones. Adriano, de hecho, los transformó poco después en un eficiente sistema de control de información ${ }^{17}$. Parece que con este emperador el cuerpo adquirió su pleno potencial, tanto en Roma como en las provincias. En cualquier caso, no estamos en condiciones de afirmar que los frumentarii perdiesen en algún momento sus atribuciones en el campo del abastecimiento y la $\operatorname{logística~}^{18}$. Se ha sugerido que estuvieron también a cargo del transporte

13. Clauss 1973, 87; Rankov 1990, 180. Véase la mención en CIL III 2063 = ILS 2370: Varronius... qui cucurrit frum(entarius) ann(os) XL. Véase también Matijevic 2014, 64-74. Su asociación con el esencial servicio de suministro y abastecimiento del ejército, en todo caso, proporcionaba una coartada notable a la hora de justificar los continuos viajes. Véase también la nota 9.

14. Livy, Epitomae, 31, 24, 4: speculator - hemerodromos uocant Graeci, ingens die uno cursu emetientes spatium; en época de César, los speculatores siempre fueron, como sugiere su nombre, soldados empleados como exploradores o "espías». Al comienzo del Principado, speculator se aplicaba a los hombres de la cohorte pretoriana adscrita a la guardia personal del emperador y que probablemente estaba compuesta en su totalidad por jinetes.

15. Dvornik 1974, 108. El emperador Macrino los usa, espiando a los legionarios, SHA Macr. 12, 4; Caracalla los usa espiando a sus amigos, Cass. Dio 78 15. Véanse también Clauss 1973, 93; Austin y Rankov 1995, 136, y Petraccia 2012, 65-88.

16. Sinnigen 1962, 211-224: "If Trajan founded the castra peregrinorum, he must have done so in the years AD 99-101». Véanse también Reuter 1999, 77-81, y Bennett 2005, 120: «[Trajan was] introducing the corps of frumentarii, officially appointed investigators drawn from the military». El último autor data estos cambios en el año 99, cuando el emperador, a su regreso a Roma, condenó a algunos de los antiguos delatores. Véanse Suet. Tit. 8, 5; Plin. Pan. 35, 1-5. Esta opción nos parece la más probable.

17. Como se relata en SHA, Hadr. 11. Para los frumentarii en su papel de informadores y observadores de lo ocurrido entre los provinciales, Sinnigen 1961, 66. Mensajeros y correos controlados por el emperador podían muy bien revelarle el contenido de cartas y documentos confidenciales. Cf. Sheldon 2006, 253.

18. Así lo defiende Menéndez Argüín 2006, 162: «El enlace entre la administración provincial de los abastecimientos y la dirección central en Roma lo proporcionarían los frumentarii, cuya sede central se situaba en los Castra Peregrina de la capital». Tácito 
marítimo y fluvial de la annona ciuica a Roma, y también se propuso que su verdadera misión era abastecer no a las legiones, sino a la propia corte imperial ${ }^{19}$.

El éxito o la importancia del cuerpo puede deducirse por su expansión posterior, ya que sus misiones se ampliaron de modo evidente, como hemos dicho. De la vigilancia y supervisión de las rutas de tránsito, esenciales para el buen funcionamiento del ejército y del Estado, pasaron a tareas de guarnición en centros urbanos y cárceles, y servicios de escolta; también aparece su presencia en algunas minas ${ }^{20}$. A la tarea de correos imperiales se unió después la recaudación de impuestos ${ }^{21}$. Los frumentarii formaron parte del sistema de policía, realizando sus funciones muchas veces con otros stationarii ${ }^{22}$. En ocasiones sirvieron como agentes del aparato de represión imperial contra los cristianos ${ }^{23}$. Finalmente, el cuerpo se convirtió prácticamente en una "policía política", aunque tal término ha de usarse con cautela en el mundo romano ${ }^{24}$. Los frumentarii viajaban por tierra y por $\operatorname{mar}^{25}$. Como legionarios, tuvieron que llevar los distintivos militares de rigor (caligae, cingulum, paludamentum), pues en ocasiones necesitaban identificarse como tales; pero en misiones realmente

ciertamente menciona unos frumentatores en el año 69, realizando misiones de búsqueda de grano (Tac. Hist. 4 35, 1), aunque su identificación con los frumentarii es conjetural.

19. Rickman 1971, 275; para la segunda teoría, Paribeni 1905, 310-320.

20. Hirt 2010, 174, 196, 201. El autor alude a los centuriones frumentarii, a los que asocia con funciones administrativas y posible gestión de minas y canteras. No obstante, de momento este tipo de soldados están ausentes en las minas auríferas del Noroeste de Hispania en el siglo II, momento en el que parecen haber alcanzado su máximo desarrollo, como demuestra la abundante documentación epigráfica al respecto.

21. Crowdy 2006, 22. Una vez que se hacían cargo de todo lo recaudado o confiscado, pudieron también guardar, vigilar y escoltar esos convoyes de grano y abastecimiento, garantizando su seguridad hasta que llegaran a destino. Como señaló acertadamente Sinnigen 1961, 69: «The Roman army in general and its secret service in particular regarded the state highway network with its way and posting stations, the military supply and taxation systems, which depended on the roads, and the various military posts which guarded them and the surrounding countryside as an integrated unit». Para Fraipoint 2019, 66-67, no obstante, la principal y más desempeñada función de los frumentarii fue la de mensajeros imperiales. En ese sentido, véase Cass. Dio 78 34, 7; 79 14, 1; 15.1; 39.3. Véanse también Clauss 1973, 87; Rankov 1990, 180; CIL III 2063, 8581 = ILS 2370, 14191. También Palao Vicente 2016, 150 y 152.

22. Los frumentarii aparecen asociados con frecuencia a las stationes del cursus publicus y del ejército, fueran esos locales los mismos para la mensajería y las tropas o se tratara de establecimientos diferentes; véanse Eck 1995, 69-70, y Kolb 2000, 160-161. También CIL VI 3329 (vía Apia), Clauss 1973, 101-105; Palao Vicente 2016, 151.

23. Rankov 1990, 181; Dvornik 1974, 107.

24. Sinnigen 1961, 65-72.

25. Aur. Víct., Caes. 39, 44. 
"secretas» seguramente actuarían bajo disfraces ${ }^{26}$. Sus antiguos cometidos, no obstante, eran proclamados orgullosamente en las inscripciones funerarias. Parece que la lanza identificadora que portaban era objeto común a los beneficiarii, signo de la autoridad y el poder que se les atribuía, en particular frente a la población civil, y no un rasgo distintivo del frumetarius $^{27}$. Este dato se complementa ciertamente con la pésima reputación posterior, y el odio generado en muchos pueblos y ciudades por sus excesos, entre los que se cuentan extorsiones, chantajes y aprisionamientos $\operatorname{arbitrarios}^{28}$. También están conectados, en ese sentido, con misiones para sofocar revueltas ${ }^{29}$.

Pero la situación profesional de estos soldados todavía alberga una gran cantidad de sombras que frenan nuestro completo entendimiento. Un frumentarius tenía acceso a promociones y traslados debido a su especialización, pudiendo promocionar en el ejército a cargos difícilmente asequibles para un simple miles gregarius ${ }^{30}$. La característica ha sido aducida

26. Sinnigen 1962, 221. Macrino, ya fugitivo (junio de 218), fue atrapado cerca de Nicomedia, mientras viajaba en carreta, pretendiendo hacerse pasar por un frumentarius para embarcarse y huir (Cass. Dio 79 39, 3 y Hdn. V 4, 11-12); esto nos indicaría algún tipo de "distintivo" $y / 0$ "uniforme».

27. Véase Perea Yébenes 1999, 269, para la aparición de frumentarii en los altares ostentando una lanza distintiva de su oficio. No obstante, Cass. Dio 78, 39 los describe sin uniforme mientras realizaban viajes. Para los objetos característicos de los beneficiarii, véase Nelis-Clément 2000, 56, 121, 282, 287: se trata de las benefiziarierlanzen, identificadoras del portador como miembro del officium consularis, rol complementario al de su pertenencia al Castra Peregrina, desempeñando la comunicación entre ambos. Véase Rankov 1990, 181-182; Sheldon 2006, 257.

28. Cass. Dio, 79 15, 1; véanse también Sheldon 2008, 12, 110, 121; Aur. Víct., Caes. 39, 44; Austin y Rankov 1995, 137; Clauss 1973, 104.

29. Austin y Rankov 1995, 137; Clauss 1973, 104.

30. Sinnigen 1961, 67: «From the point of view of their careers as well as of their recruitment, the frumentarii were typical enlisted men. That is to say, they had very little chance of rising high in the military or administrative hierarchy. Some held other administrative posts as non-commissioned officers before death or retirement; some reached the centurionate, but few of them who rose from the ranks were promoted into the equestrian hierarchy of office-holding. The chances for speedier advancement were greater if a man had been commissioned a centurion directly upon recruitment and had served as frumentarius in that rank. In such a case fast advancement probably depended more on his having begun as a centurion at an early age than on his having been employed in the secret service». Se conocen promociones directas al centurionado: CIL XIII 6682 (Mogontiacum); AE 1905, 25, y AE 1930, 151 (Emérita Augusta); Clauss 1973, 110. También indirectas, desde el puesto de optio: CIL XI 1322; Clauss 1973, 110-111. Se asume que pudieran ascender a los speculatores, con los que están estrechamente asociados: CIL III 3524 (Aquincum) y CIL III $14479=$ AE 1901, $154=$ IDR III/5 426 (Apulum). En casos excepcionales, pueden beneficiarse del favor imperial para llegar a los rangos más altos de praefectus praetorio, praefectus Urbi o incluso cónsul: Clauss 1973, 113. Según Sinnigen 1961, 68, «Only during the Severan 
recientemente por McCunn para dudar del papel de los frumentarii como soldados dedicados expresamente al «espionaje»: el problema de la revelación de secretos hubiera sido ciertamente desastroso cuando se cambiase de puesto o destino. El mismo autor defiende que la principal función de los frumentarii continuó siendo el suministro, y que, conforme el siglo III avanzaba, las requisas extraordinarias que los diversos emperadores fueron decretando para abastecer a las tropas, en tiempos en los que el estado de alarma, como es sabido, era casi constante, les fueron abriendo paso a un rosario de diversas funciones ${ }^{31}$. McCunn observa además otros inconvenientes respecto a la posible ocupación de los frumentarii como "agentes secretos": los gobernadores, aunque es sabido que los tenían en sus séquitos, no confiarían en ellos, conociendo su profesión e intenciones potencialmente peligrosas para sus intereses. Este problema no obstante desaparece si consideramos que pudieron desempeñar su labor usando identidades falsas, o permaneciendo ocultos (esto es, los espiados no eran conscientes de serlo; tal es la manera más eficaz de recabar información

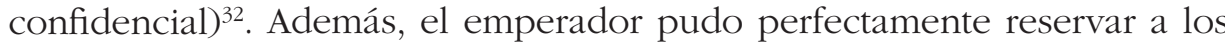
frumentarii para otros cometidos: existían muchos servidores en el Imperio, y por lo tanto mil maneras distintas de espiar sin levantar sospechas. Está demostrado que las mismas funciones que desempeñaban los frumentarii la hacían distintos soldados, no siempre officiales ${ }^{33}$.

Otra opción, posible, aunque difícil, es que un frumentarius no desvelase su ocupación, salvo ante el mismo emperador o en situaciones críticas, aunque su hoja de servicios fuese puesta al descubierto después, tras la honesta misio, y figurase en los epitafios. En cualquier caso, los datos apuntan a un cuerpo de estructura flexible e informal; el tan controvertido rol de «espías» no sería una ocupación fija, ni mucho menos obligatoria. En

dynasty do former secret service agents, and these rather few, attain the highest honors as praetorian prefects, senators, or even, in one case, as consular colleague of the emperor». Para M. Advento, véase Cass. Dio 79, 14, 3.

31. McCunn 2019, 5. La misma opinión en Sheldon 2005, 251.

32. Para algunos autores, estos individuos no ocupaban el puesto de frumentarius una vez introducidos en el equipo de gobierno local. Así, Mann 1988, 149: "Although it has been claimed that frumentarii served as such in the officia of provincial governors, there is no evidence for this - even though they are sometimes associated with members of provincial officia on inscriptions. On the contrary, it is now clear that when transferred into a governor's officium, they no longer ranked as frumentarii.. Esta afirmación fue matizada por Rankov 1990, 179-180. Un gobernador era, esencialmente, también otro "hombre del emperador».

33. McCunn 2019, 7; Bingham 2012, 13-14 y 94. 
definitiva, como afirmó W. G. Sinnigen, estos soldados mostraban notables diferencias con los servicios secretos actuales ${ }^{34}$.

\section{ESTRUCTURA}

Los frumentarii permanecían registrados en las legiones de las provincias, aunque sometidos a una cadena de mando distinta. Para algunos estudiosos esta pertenencia a una legión era una mera formalidad administrativa; otros, como N. B. Rankov, defienden que se trató de una característica de vital importancia ${ }^{35}$. En Roma formaban el numerus frumentariorum, con sus propios centuriones y oficiales subalternos ${ }^{36}$. Estos frumentarii seguían perteneciendo a sus legiones de origen pese a estar concentrados en la capital. Servían bajo el mando de un centurión primus pilus, oficial que solía ser referido como princeps peregrinorum, por la procedencia de sus tropas ${ }^{37}$. En el monte Celio, lugar de su cuartel, se encontraban doce o trece soldados de cada legión. Una serie de inscripciones dedicadas por tales soldados, en este sentido, honran el genio del lugar donde permanecían acuartelados (Castra Peregrinorum) ${ }^{38}$. Posiblemente el número total de frumentarii alcanzase varias centenas,

34. Sinnigen 1961, 65: «Actually, the resemblance between the Roman internal security police force and its modern counterparts, while close in some important particulars, is by no means complete. Accordingly, the expression «secret service» should be conceived as a convenient, if not perfectly descriptive, label for an ancient institution which has no exact modern parallel». Véanse también Liberati y Silverio 2010, 111-116; Sheldon 2006, 255; Kagan 2011, 161-173.

35. Mann 1988, 150, se decanta por la primera opción, y Rankov 1990, 178, por la segunda. Véase Cupcea 2009, 307: "Although it has been thought that only the legions of the north and western armies were providing these officiales, epigraphical evidence indicates that, coming from every legion of the Empire, the frumentarii gather at the Capital, in a numerus frumentariorum, an ad-hoc military unit, organized in Rome for the emperor's personal use».

36. Austin y Rankov 1995, 136; Cupcea 2009, 306; Fraipoint 2019, 59. Véase también Faure 2003, 377-427, y Busch 2007, 315-341.

37. Reynolds 1923, 168-189. Un centurio frumentarius de hecho podía ascender y convertirse en un primus pilus legionis, CIL VI 423, 1636, CIL X 6657; Clauss 1973, 112-113. Eso solía ser el cénit de su carrera profesional. Véase también Panciera 2006, 1453-1464.

38. Rankov 1990, 176-182. Coarelli y Usai 1984, 180. Véase también Perea Yébenes 1999, 187: "Castra Peregrina, donde se dan cita frumentarii de todos los rincones del Imperio", y Perea Yébenes 2020, 6: Diversos estudios han demostrado que los «servicios secretos" militares se organizan desde Roma, en los llamados castra peregrina/peregrinorum situados en el Celio. Los soldados que hacen esta labor se denominan frumentarii, y son, en general, centuriones, que se desplazan a las provincias para obtener o transmitir información «secreta». Véanse también Panciera 2006, 1471-1482; Sheldon 2006, 250-260. 
teniendo en cuenta que una cantidad similar a la establecida en Roma debió hallarse repartida por otras capitales provinciales en un momento dado. En Roma, probablemente obedecían órdenes del Prefecto del Pretorio $^{39}$. La distribución y el número total de los frumentarii han sido usados a menudo como método para tratar de averiguar tanto el origen social como las verdaderas funciones del cuerpo ${ }^{40}$. Existía, como es comprensible, un considerable movimiento de estos soldados, que se desplazaban a sus provincias (los destinos de origen) y luego regresaban por algún tiempo a la propia Roma. Curiosamente, la legión II Pártica, estacionada a muy pocos kilómetros de la capital, no nos ha dejado frumentarii documentados. Los soldados enviados a la Urbs por asuntos de su gobernador eran conocidos colectivamente como peregrini, de ahí su acuartelamiento y su fuerte vinculación al Castra Peregrina ${ }^{41}$.

Para algunos autores, los frumentarii debían sus ascensos y promociones al emperador ${ }^{42}$. No obstante, otros investigadores, apoyados en la epigrafía e interpretaciones de las fuentes, difieren, al haber casos de frumentarii sirviendo directamente bajo las órdenes de un gobernador ${ }^{43}$. En cualquier caso, los frumentarii eran distintos a los demás miembros del servicio público romano del Alto Imperio. Se podría hablar quizás de una doble lealtad, al poder imperial y a los distintos gobernadores de las provincias, aunque la propuesta de interpretación que mostramos anteriormente de sus funciones podría resolver estos inconvenientes. Parece,

39. Se habla de cien hombres en Roma, al mando del Prefecto del Pretorio (Sinnigen 1962, 221-224). Los frumentarii de las provincias estarían a las órdenes directas de un legatus legionis según Perea Yébenes 1999, 236-237. Para Crowdy 2006, 22, serían doscientos los frumentarii de Roma. Véase la nota 9.

40. Forni 1953, 45 y ss., destaca la gran variedad de ambientes sociales de los que procedían, desde clases humildes a familias de provinciales ciertamente acomodados, como el caso de Elio Marcelo (CIL III 1182).

41. Se sabe que al menos dieciséis frumentarii fueron enviados desde Roma a la sede de su legión o al officium consularis de su provincia de origen. En Hispania, los frumentarii son enviados directamente al officium consularis de Tarraco, junto con los speculatores, commentarienses o cornicularii, aparentemente sin su etapa de preparación en Roma. Véanse CIL II 4150, 4154, 4170, 6088; Clauss 1973, 96; Rankov 1990, 180; Menéndez Argüín 2003, 317.

42. Fuhrmann 2012, 151-157. Su estrecha conexión con el emperador es revelada por la expresión frumentarius Augusti, mencionada en las inscripciones (IGRR III $80=$ ILS 9476, AE 1977, 60, ILS 9473, CIL XIII 1771); véanse también Mann 1988, 150; Rankov 1990, 177; Austin y Rankov 1995, 136. No es diferente del frumentarius legionis, pero está sirviendo en una misión especial directamente bajo órdenes del soberano.

43. Austin y Rankov 1995, 151-152. 
en cualquier caso, que sus tareas para el soberano y los gobernadores fueron, en muchos sentidos, complementarias, y no antagónicas ${ }^{44}$.

\section{LOS FRUMENTARII: SOLDADOS ESPECIALISTAS}

Eran las legiones las que elegían y mandaba a sus frumentarii a Roma, por lo que algunas cualidades deberían distinguir a estos soldados ante los ojos de sus oficiales y mandos. Los frumentarii como legionarios, al menos inicialmente, tuvieron que recibir el mismo entrenamiento básico que viene caracterizado en las fuentes literarias ${ }^{45}$. Además, dados sus cometidos, parece claro que se trataba de soldados adiestrados en el subterfugio y la observación, siendo capaces de desenvolverse al aire libre y en las diversas técnicas de engaño: suplantación, infiltración, camuflaje, etc. Consideramos probable que en las regiones fronterizas también contasen y comprobasen el número de adversarios, como exploradores militares, con los que aparecen muy unidos ${ }^{46}$. Además, su empleo a la hora de asesinar a personajes importantes, que como veremos parece generalizado a finales del siglo II, incluiría las habilidades para esconderse, disfrazarse y/o escapar ante una inminente persecución. Estas técnicas, de modo global, indicarían un cuerpo de soldados con marcadas habilidades diferenciales ${ }^{47}$.

44. Fraipoint, 2019, 15: À l'instar des empereurs, les gouverneurs, dès le début du Principat, ont employé des militaires pour communiquer avec Rome. Même les gouverneurs des provinces sénatoriales, qui ne possédait donc pas une armée importante, disposaient de troupes à cet effet. Les soldats, envoyés dans l'Urbs pour les affaires de leur gouverneur, étaient connus collectivement sous le nom de " peregrini " ou "étranger ".

45. Veg. Mil. I 8-18: nadar, montar, tiro con arco, manejar la lanza y espada, practicar la marcha pírrica, atletismo, etc. Según Perea Yébenes 1999, 238, «el rango de los frumentarios hay que situarlo entre los suboficiales de mayor rango y antigüedad, pues son mencionados reiteradamente junto a los specuatores y a los centuriones. Así sucede en inscripciones de colegios militares en las provincias». Vegecio también nos recuerda que los soldados legionarios instruidos en habilidades de gran valor obtenían doble paga y doble ración (Veg. Mil. II 7). Como hemos visto, podría ser el caso de los frumentarios.

46. Perea Yébenes 1999, 235: muchos servían en legiones estacionadas permanentemente en las fronteras, aunque viajasen a Roma. Dentro de los officia provinciales, los frumentarii compartían funciones con los speculatores y beneficiarii y, en algunos casos, incluso con los commentarienses. Así, CIL XIII 1771 (Lugdunum). Véanse Clauss 1973, 98; Speidel 1983, 63-78; Nelis-Clément 2000, 133-210. También CIL VI 3357, 3359: un speculator del ejército romano en Britania conmemorado por un frumentarius de la legión XX Valeria Victrix, también estacionada en la isla. Véase también Rankov 1987, 243-249 y n. 30.

47. Martini 1980, 143-151, habla de mensajeros y «espías» con Adriano, y bajo Caracalla, actuando como asesinos (afirmación errónea, pues están atestiguados como asesinos desde 
¿Dónde y cuándo recibían los frumentarii tal instrucción específica? A buen seguro en Roma ${ }^{48}$. Allí están atestiguados, en ocasiones, como entrenadores de otras unidades de elite, por lo que debemos considerar que se trató de elementos especializados en una serie de conocimientos o técnicas a gran nivel ${ }^{49}$. Dados sus fuertes lazos con las provincias, es posible que también reflejasen las costumbres bélicas o prácticas militares relacionadas con determinados pueblos y que hubiesen podido permanecer, aun en forma residual, dentro del Imperio, especialmente en las fronteras (Britania, Galia, Asia, etc.). No obstante, no se trató nunca de un numerus compuesto por hombres provenientes de provincias o territorios específicos, destinados a luchar en el campo de batalla con tácticas y armamentos propios $^{50}$. Pero para soldados que debían cumplir misiones de infiltración $\mathrm{y}$ «espionaje», el desenvolverse bien en las zonas fronterizas y militarizadas debió convertirse en requisito esencial. Esto sucedió a menudo durante la historia del ejército, y resultó común con las diversas unidades formadas en mayor o menor número por elementos de raigambre germánica a lo largo del tiempo ${ }^{51}$. Recordemos que para F. Dvornik los enigmáticos arcani o areani presentes en el área fronteriza de Britania pudiesen haberse tratado de frumentarii, al menos en su origen. Dadas las múltiples funciones del cuerpo y sus evidentes similitudes con los agentes que

el año 182 al menos). El autor los relaciona con "el mantenimiento del orden público", y destaca que su reclutamiento alberga parecidos con los de los speculatores y equites singularis legionis. Esta vinculación, muy repetida en la historiografía, nos hace pensar que los frumentarii mantuviesen también, quizás todo el tiempo, competencias de exploración militar.

48. Clauss 1973, 83; Rankov 1990, 177.

49. Speidel 2002, 53, con nota. Una sola inscripción evoca un exercitator frumentariorum, que nos permite deducir que los frumentarii eran tropas montadas, algo lógico, por otra parte, para soldados tan móviles. Véase CIL VIII 1923. Un centurio frumentarius podía desempeñarse como exercitator singularium Imperatoris: CIL VIII 2825 (Lambaesis); también Clauss 1973, 112. Speidel 1993, 34, 753, 754, 755, documenta frumentarii que sirvieron como entrenadores de la Guardia Imperial.

50. Su papel asociado a las zonas fronterizas debe matizarse. Fuera de Roma e Italia parecen relacionados con el orden público, pero también las comunicaciones e información. En el caso de Lyon, la provincia no cuenta con una guarnición de legiones permanentes, aunque sí una cohorte urbana, lo que resulta de por sí una rareza (único caso junto a Roma y Cartago). Los frumentarii en cambio estaban presentes en la ciudad. En Hispania, los frumentarii también están documentados; pese a no haber fronteras, existían, como es sabido, fuerzas militares. Véanse Padilla Monge 1989, 18, y Bérard 2015, 365-381.

51. Por ejemplo, los bátavos eran afamados por guerrear con destreza cerca y dentro de los ríos, desde los tiempos de Julio César (Caes. BGall., IV 10). Muchos de estos soldados tenían un trasfondo completamente local. Así, Perea Yébenes 1999, 271: "procedentes de las provincias, llegaban a la capital para recibir consignas o para facilitar información de interés estratégico al propio emperador». 
controlaban el territorio como exploradores y ofrecían inteligencia militar, informándose del ánimo e intenciones de los pueblos bárbaros más allá del limes, no nos resulta descabellada tal hipótesis ${ }^{52}$. A este rol, como veremos a continuación, se unió otro, muy significativo, relacionado con sus habilidades militares y de infiltración: el de neutralizar a objetivos o enemigos concretos por orden del emperador.

\section{LOS FRUMENTARII COMO FACTOR BÉLICO DECISIVO PARA ELIMINAR ENEMIGOS CLAVE}

Durante los reinados de Antonino Pío y Marco Aurelio la actividad de los frumentarii tuvo que continuar, pero sus funciones comenzarán a expandirse con la llegada del último gobernante de la dinastía, Cómodo. En algunas de sus expeditivas medidas de gobierno se valió de los frumentarii: así, los utilizó para eliminar al bitinio Saotero, chambelán de palacio, y puede que también con Tigidio Perenio, prefecto del Pretorio asesinado en el año 185, emplease a estos soldados ${ }^{53}$.

Tras la muerte del propio Cómodo la última noche del año 192, se produce una grave conmoción en la ciudad ${ }^{54}$. Publio Helvio Pértinax recibió el apoyo del pueblo y el Senado, que lo proclamó con un decreto. Pero se granjeó la enemistad de los partidarios de su predecesor, y fue asesinado al poco tiempo. Tras ello, los soldados de la ciudad realizaron una subasta en el campamento, para vender la corona imperial al mejor postor. El elegido fue Marco Didio Juliano ${ }^{55}$. Pero las noticias de los confusos acontecimientos en la capital imperial se extendieron pronto por las provincias, lo que fomentó el clima de agitación. En Panonia, Lucio Septimio Severo se erigió hábilmente como el vengador de Pértinax, que era idolatrado por las legiones locales; así, pudo ganarse la confianza de tropas muy numerosas y de una excelente calidad combativa. Con la misma sagacidad, se asoció a su potencial rival, Clodio Albino, al que designó César ${ }^{56}$.

52. Dvornik 1974, 117. Véanse también Birley 1966, 35-42; Southern 1989, 84; Lee 2006, 179; Sheldon 2006, 164-176; Amm. Marc. 28, 3, 8.

53. Hdn. I 9, 8-10; el modo en que los hijos de Perenio fueron buscados, apresados, trasladados y ejecutados por el camino casa muy bien con el modus operandi de los frumentarii. Véanse también Dvornik 1974, 103; SHA, Comm. 4, 5.

54. Birley 1969, 247-280.

55. Tras prometer grandes sumas y ricos regalos, fue proclamado por los pretorianos y escoltado al palacio imperial. Véase Appelbaum 2007, 198-207.

56. En Britania, por otra parte, ya se manifestaba un cierto descontento entre las legiones desde años atrás. Décimo Clodio Albino, al mando en la zona, se siente seducido 
El gobernador de Siria, Pescenio Níger, decidió también proclamarse emperador, pero permaneció en sus propios dominios, esperando aglutinar el apoyo de las ricas provincias orientales. Sabemos que mandó cartas y edictos al Senado y al pueblo de Roma, donde parece que gozó de simpatías. Pero Severo interceptó tales misivas por medio de unos "emisarios", que mueven poderosamente a pensar en los frumentari ${ }^{57}$. Como hemos dicho, se utilizaban frecuentemente como mensajeros o correos, así que, si era posible ganar su fidelidad, los servicios prestados a cambio podían ser de excepcional importancia y decisivos. Un frumentarius fue también enviado desde Roma por Didio Juliano para que asesinase a Níger en su capital, Antioquía. Esto prueba tanto el nerviosismo de Juliano como las habilidades atesoradas por estos soldados especiales, y, por último, la no pequeña importancia de Pescenio Níger en la escena política ${ }^{58}$.

Plenamente consciente de su delicada posición, Juliano recurrió nuevamente a los frumentarii, para intentar eliminar a otro de sus rivales, un suceso que conocemos gracias a pasajes de las fuentes literarias que a nuestro juicio no han recibido la atención suficiente. Así, envió a «Aquilio, el asesino de senadores", con la misión de acabar con Septimio Severo y abortar de tal modo el avance de las legiones panonias contra Roma ${ }^{59}$. Inicialmente el personaje acepta el encargo y marcha a su cometido; el epíteto indica que su reputación tuvo que producirse tras una serie de éxitos en tales asuntos, ciertamente delicados. Creemos, por tanto, que habría recibido un entrenamiento muy marcado, que seguramente resultó decisivo a la hora de propiciar su controvertida fama. Recalcaremos de nuevo que un individuo calificado como "asesino de senadores» o «asesino de generales» nos hace pensar en alguien perteneciente a un cuerpo con funcionamiento distinto al de las unidades regulares de infantería de aquel tiempo, cuyo entrenamiento básico no incluiría la preparación para tales menesteres especiales ${ }^{60}$. El principal biógrafo actual de Septimio Severo, A. R. Birley, también recoge el episodio, dedicando algo de atención a M. Aquilio Félix, aunque, como veremos, mostrando dudas ${ }^{61}$. Otra de nuestras

por la idea del poder imperial, al alcance de su mano. En Roma contaría igualmente con una facción del Senado, que apoyaría eventualmente su proclamación, pero esta no llegó a producirse por su alianza con Severo. Véase van Sickle 1928, 123-127.

57. SHA, Sev. 6, 7-8.

58. Véase SHA, Sev. 5,8

59. SHA, Did. Iul. 5, 8. CIL X 6657 = ILS 1387; Hdn. III 5, 4-5; Clauss 1973, 91; Rankov $1990,176$.

60. SHA, Pesc. Nig. 2, 6.

61. Birley 2012, 152, y McCunn 2019, 4: «The Historia Augusta does not identify him as a frumentarius, but an inscription found at Antium records a certain Aquilius Felix who 
fuentes literarias para el episodio, Dion Casio, no solo menciona al citado agente, sino que indica que Juliano mandó a más soldados, semejantes al anterior ${ }^{62}$. Aunque la Historia Augusta muestra pesimismo ante la idea de enviar hombres aislados contra pretendientes muy importantes, protegidos por miles de legionarios en campamentos o palacios repletos de armas y guardias, lo cierto es que tanto anteriormente en Grecia como en el propio Imperio, y después en época medieval, tales cometidos aparentemente desesperados podían triunfar: un hombre intrépido, experto, decidido y bien entrenado, que use la confusión y pase desapercibido entre una gran multitud, especialmente si se desconoce su llegada, quedaba capacitado para intentar al menos tamaños objetivos ${ }^{63}$.

Las fuentes nos informan, no obstante, que estos individuos fueron pasándose al bando de Severo. Pensamos que el verse irremisiblemente asociados a un régimen sin proyecto político, que a todas luces iba a resultar derrocado por su incapacidad manifiesta de lidiar con las inevitables represalias de las aguerridas legiones danubianas, fue el motivo principal que hizo cambiar de opinión a esos frumentarii; aunque la dificultad de la misión no resultaba ni mucho menos pequeña, en ese tiempo ya estaban acostumbrados, probablemente, a llevar a cabo cometidos ciertamente llenos de riesgos. Pero tuvo que imponerse la sobria realidad y el sentido práctico ${ }^{64}$.

Tras su victoria, acudiendo al Senado con amigos y rodeado de guardias, Severo explicó después que había tomado el poder para defender su vida, puesto que el anterior emperador «había enviado contra él para matarle a unos individuos que ya eran célebres por haber asesinado a generales ${ }^{65}$. En la rápida conquista de la capital, las fuentes nos hacen

was active as a centurio frumentarius before being promoted to a procuratorship and then praefectus classis prouinciae Rauennatis». Véanse también James 1946, 311-319, y Sheldon 2008, 253.

62. Véase Cass. Dio 74 16, 5: «Y él [Didio] continuó enviando hombres contra Severo para asesinarlo a traición".

63. Recuérdese, en circunstancias similares, el propio caso de Amiano Marcelino, introducido por Augusto Constancio II en un equipo especial cuyo fin era llegar a Colonia en el año 355 y eliminar al usurpador Silvano. Aunque el episodio narrado tenga evidentes puntos oscuros, resulta un ejemplo válido para nosotros por los paralelos con lo acontecido a Clodio Albino. Véanse Amm. Marc. 15, 5; Nutt 1973, 80-89. Hay muchos ejemplos medievales; por ejemplo, véase Martines 2003, 111-173, para los Pazzi en 1478 y el planificado intento de esa familia de banqueros para acabar con los Medici en la catedral florentina. En Grecia, no obstante, esas misiones tenían mucho de suicidio: véase Penwill 2010, 24-52.

64. Así, SHA, Pesc. Nig. 2, 4. Véase también SHA, Did. Iul. 5, 1. De tal modo, los enviados se fueron pasando al bando de Severo; véase también Cass. Dio 74 17, 1.

65. SHA, Sev. 7, 4. Véase también Rantala 2016, 160. 
pensar que Severo controlaba otras unidades de tropas especiales, sin que podamos especificar si eran frumentarii o no. Así, soldados con armas escondidas y vestidos de campesinos y arrieros se infiltraron en Roma, ocupando los caminos y otros puntos vitales en los alrededores ${ }^{66}$. Del mismo modo, Severo envió un grupo de hombres que se apoderó del Castra Praetoria, "por caminos y senderos diferentes" ${ }^{67}$.

No obstante, su uso de estas tropas en misiones diferenciadas puede que no termine aquí. Severo, según se cree, habría decidido eliminar a Clodio Albino desde el principio, pues lo veía como una molestia, necesaria al comienzo de la guerra, para mantener su retaguardia cubierta; una vez que hubo terminado con Níger, la labor de dicho «aliado de circunstancias» había concluido. Albino tuvo que entender al fin que había sido utilizado, y se puso en pie de guerra, pese a su inferioridad numérica. Severo había servido en la Galia durante su larga carrera política, y tuvo que conocer el carácter aguerrido de sus habitantes: las legiones occidentales serían un desafío formidable, incluso para sus reputadas tropas ilirias. Por lo tanto, ideó una estratagema para deshacerse de su rival sin tener que jugarse el todo por el todo en una batalla: decidió que, fingiendo una embajada confidencial, sus hombres asesinasen a Albino mientras estaban a solas con él ${ }^{68}$. Pero este desconfió, cuando los mensajeros le pidieron reunirse en privado para comunicarle un secreto. Los emisarios fueron apresados, y se les descubrieron armas escondidas; entre torturas, finalmente admitieron haber sido enviados por Severo para matarle ${ }^{69}$. Todo el contexto de la situación nos hace pensar en los frumentarii como elegidos para llevar a cabo tal hecho.

Como se puede apreciar, ante una situación de enorme tensión, con varios contendientes deseosos de alcanzar el poder supremo y los ejércitos provinciales desplegándose para la guerra, empieza a acentuarse de modo notable el perfil más militar de los frumentarii, como soldados especiales cuyo entrenamiento les permite actuar en difíciles misiones. Por lógica tuvieron que estar presentes en todos los bandos mencionados,

66. Hdn. II 12, 1.

67. Hdn. II 13, 12. Vemos que tales ardides estaban presentes en los tratadistas militares griegos anteriores, como Onasandro (Strat. XXXIX 4-7) y el más antiguo Aen. Tact., XXIX 1-11.

68. Birley 2012, 186, a nuestro entender sin argumentos, opina que el hecho es inverosímil, y lo califica de invención. Observemos en cambio cómo, según el relato, los enviados de Severo pidieron hablar con Albino a solas, y no intentaron matarlo delante de sus tropas o guardias, lo que hubiese acarreado su ejecución inmediata y muy probablemente el fracaso de la misión, además sin posibilidad de escapar.

69. Hdn. III 5, 4; Rodríguez González 2010, 86. Véase también SHA, Clod. 8. 
pues existieron en cada legión, salvo al parecer en la II Pártica, como hemos dicho.

Una vez se vio como señor del Imperio, Severo procedió a implantar sus controvertidas reformas militares. En ellas se incluyó el aumento de importancia y número de los frumentarii, prueba segura de los buenos ojos con los que el emperador juzgaba los servicios del cuerpo, y un argumento indirecto acerca de la valía probada en las guerras civiles. Es posible que, en ese clima de inestabilidad y desconfianza, el tipo de habilidades presentadas por estos soldados especiales resultasen valiosas en extremo, sobre todo si su fidelidad había quedado garantizada ${ }^{70}$.

A Severo le sucedieron sus hijos en 211, Geta y Caracalla. La intensa rivalidad entre los hermanos acaba con el asesinato de Geta en 212. Tras la muerte de Caracalla en 217, seguimos teniendo noticias del uso de los frumentarii para controlar la disidencia y obtener información confidencial en el reinado de su sucesor, Macrino ${ }^{71}$.

En la sublevación senatorial contra Maximino del año 238, los frumentarii fueron importantes a la hora de privarle de suministros durante su infructuosa invasión de Italia. Así, tras guarnecer en plazas fuertes, graneros fortificados y ciudades amuralladas las cosechas, ganados y alimentos, el ejército del emperador pronto se vio sumido en penalidades, que fomentaron el descontento y quizás aceleraron el asesinato de Maximino junto a su hijo y heredero, Máximo. Este papel decisivo en tal enfrentamiento nos mueve a pensar que el cuerpo realmente no abandonó sus tareas relativas al abastecimiento y control de las vías de comunicación, o incluso de escolta y vigilancia de abastecimientos y suministros ${ }^{72}$.

70. Véase Perea Yébenes 1999, 363: «Nunca un emperador tuvo tanto poder militar en su mano. A ello se añade la potenciación del cuerpo de frumentarii en los castra peregrina de Roma. Estas unidades tienen una extraordinaria importancia, pues encuentra en los hombres destinados a la statio verdaderos vehículos de trasmisión de ideas, de creencias o de instituciones entre Roma y las legiones provinciales (y viceversa). Simultáneamente a cambios tan importantes en la guarnición de Roma, Severo sitúa en las provincias militarizadas a hombres de su plena confianza curtidos en la guerra civil; y se crean numerosos cuerpos de caballería móviles».

71. SHA, Macr. 12, 4. Véase la nota 15.

72. SHA, Max. et Bal. 10, 3. Consideramos innecesarias las trabas puestas a este fragmento por Clauss 1973. En este sentido, CIL VI $230230=36748=$ ILS 2216 (Roma): frum(entarius) leg(ionis) XIII Gem(inae). M. Aurelius Sophaenetus, designado para una misión en una statio de la Via Appia. Allí, junto a su colega Q. Haterius Valerianus, de la Legión VIII Augusta, erigieron un altar al emperador Severo Alejandro (222-235), al genius del castra peregrinorum y a todo el ejército. Control de puertos: CIL XIV 7, 125 (Ostia) y CIL X 1771 (Puteoli); Clauss 1973, 82, 90. 
Los frumentarii desde entonces siguieron siendo utilizados, e incluso parece que en los convulsos años de la Anarquía Militar su actividad se multiplicó, muy posiblemente en conexión con las difíciles situaciones atravesadas por el Imperio; así, su labor resultaría irremplazable en las requisas forzosas y extraordinarias que se sucedieron durante muchos $a^{n}{ }^{73}$. En ese contexto es cuando se muestran con más fuerza los abusos y conflictos con los provinciales de los que hemos hablado anteriormente. Dada la gran parquedad de nuestras fuentes para el periodo, tampoco podemos descartar que los frumentarii estuvieran involucrados, como brazo ejecutor, en al menos algunos de los numerosos asesinatos de emperadores y usurpadores en la segunda mitad del siglo III.

Tanto Birley como más recientemente McCunn muestran dudas respecto a las citadas informaciones sobre el empleo especial de los frumentarii, principalmente por pertenecer a la Historia Augusta, fuente que con razón consideran de poca confianza. Nosotros creemos que en esta ocasión los miedos son injustificados ${ }^{74}$. El mismo McCunn remite a Ronald Syme como autoridad de referencia respecto al tema, pero quizás no tuvo en cuenta que Syme siempre consideró que la fuente mayoritaria empleada hasta el año 217 era excelente, sobria y fiable ${ }^{75}$. Es el material empleado en las nueve biografías calificadas por T. Mommsen como principales, y ocupa casi la totalidad del texto, salvo unos pequeños aderezos de carácter malicioso, posiblemente procedentes de Mario Máximo, y que en todo caso no afectan a nuestros pasajes ${ }^{76}$. Además, las noticias están corroboradas por los testimonios paralelos e independientes de los contemporáneos Dion Casio, que atravesaba por aquel entonces momentos decisivos en su carrera política, en la que llegó a cónsul años después, en 229, y Herodiano. Creemos que este aspecto es vital, pues el senador griego, pese a no participar en la guerra civil, tuvo que estar bien informado en lo referente a sucesos esenciales en el devenir histórico del

73. SHA, Claudius Gothicus 17, 1 (donde aparecen bajo órdenes del emperador Galieno espiando al futuro Claudio II). Véase también McCunn 2019, 12-13, y Perea Yébenes 2017, 1-34.

74. McCunn 2019, 3. Se trata de informaciones a menudo complementadas o confirmadas por otras fuentes. Sería interesante, en trabajos futuros, estudiar la relación literaria de los frumentarii con los autores latinos unidos a la tradición de la Kaisergeschichte pues de allí parece proceder la tradición.

75. Syme 1972, 287-302, y 1983, 12-29.

76. Syme 1968, 91: «He [Marius Maximus] used the Autobiographies of Hadrian and of Severus, but with alert and insidious criticism. That is clear from the fragments preserved in the HA. And he was eager to bring out the worst». También se usó el buen material en partes de las vitae de algunos pretendientes. 
momento, que como sabemos le inmiscuían de manera directa ${ }^{77}$. Por estas razones diferimos del excelente trabajo de McCunn en este sentido, ya que elude profundizar en la vertiente militar del cuerpo, especialmente en la importancia de los frumentarii respecto a los numerosos asesinatos políticos en los que fueron empleados en periodos de máxima tensión. Sin descartar que, como afirma, las tareas de abastecimiento y distribución permaneciesen dentro de las atribuciones directas de los frumentarii, o que incluso fueran las más importantes, para nosotros queda claro que, a diferencia de otros puestos, estos soldados mantuvieron una gran preponderancia principalmente por su entrenamiento y habilidades bélicas, que en ningún momento se perdieron, según acabamos de mostrar. En cambio, en ese periodo de transformaciones políticas y sociales que empieza con el siglo III, vemos también cambios muy importantes dentro del ejército, en los que incluso algunos rangos y puestos anteriores se desvinculan completamente de su función militar ${ }^{78}$.

\section{Conclusión}

Los frumentarii, ocupados del control del suministro del ejército e integrantes después de un servicio de mensajería y correos imperiales, eran también, a finales del siglo II, legionarios distintos y distintivos, preparados para realizar misiones especiales que incluían la infiltración, la suplantación, el «espionaje» y, cuando situaciones muy graves lo requerían, el asesinato político. No cabe duda de que, pese al progresivo cambio de rol, los frumentarii siempre se mantuvieron como individuos fiables a la hora de llevar a cabo una serie de cometidos peliagudos, para los que estaban preparados gracias a un buen manejo de información, facilitado por su labor frecuente de mensajeros, y más concretamente por su entrenamiento específico. Como indicó W. G. Sinnigen, a menudo estaban encargados de realizar «el trabajo sucio", y sabían cómo hacerlo. Creemos haber demostrado que su preponderancia en el Imperio y las numerosas funciones realizadas, que se fueron ampliando a lo largo del tiempo, se

77. Véanse Millar 1964, 17 y 27, que nos recuerda la obra sobre la guerra civil de 193-197 escrita por Dion Casio y desgraciadamente perdida; también Rantala 2016, 160, que atribuye validez al testimonio de la guerra entre Severo y Juliano de la Historia Augusta, y Kemezis 2020, 257-286.

78. Los frumentarii mantuvieron sus capacidades militares, el entrenamiento y las habilidades de legionario hasta el final, a diferencia de otros; véase por ejemplo Cowan y McBride 2003, 12-13: el antaño determinante primus pilus se convierte en un cargo fiscal, luego se desvincula del ejército y, por último, desaparece. 
debían, al menos en una parte importante, al entrenamiento militar específico. Soldados de carácter tan móvil muy posiblemente fueron, como ya hemos indicado, tropas montadas, lo que refrendaría y recalcaría en todo momento su rol y estatus, y una idiosincrasia particular dentro del ejército romano, en unos momentos en los que estaban tomando carta de naturaleza estas agrupaciones de soldados de elite ${ }^{79}$.

Esta situación se prolongó, con intensidad cada vez mayor, hasta el fin de las convulsiones del siglo III y la restauración del orden con la Tetrarquía. Preocupado, según se cree, por la siniestra fama atesorada por los frumentarii, el emperador Diocleciano disolvió el cuerpo ${ }^{80}$. Pero pese a las connotaciones negativas asociadas durante siglos a los frumentarii, el denominado "Nuevo Imperio", marcadamente autocrático, no podía permitirse el prescindir de semejantes servicios, por lo que muy pronto los agentes in rebus ocuparon su lugar, realizando idénticas funciones y cometiendo también los mismos abusos ${ }^{81}$. McCunn, por otra parte, ofrece una hipótesis plausible a la disolución de los frumentarii que no está relacionada con su sórdida reputación; simplemente Diocleciano dejó de necesitarlos tras la creación, o la culminación, de un cuerpo centralizado y más eficaz, sin conexiones con una legión o provincias específicas, una vez que las anteriores esporádicas requisas fueron reguladas y estandarizadas en la annona militaris, dejando de tener carácter extraordinario $^{82}$. Así, el perfeccionamiento de un nuevo sistema de tasación los haría redundantes e innecesarios. Con este modelo, los suministros para el ejército se materializaban a través de impuestos en especie recaudados por funcionarios. Un censo detallado hacía que las cantidades necesarias para sufragar los impuestos se planificasen con antelación, siendo conocidas de antemano en las distintas aldeas, pueblos, etc. Por tanto, la implantación de la annona pudo resultar directamente proporcional a la disminución del rol de los frumentarii. Los agentes in rebus irían adueñándose así, progresivamente y en los tiempos siguientes, de todas las otras funciones desempeñadas por sus antecesores.

En cualquier caso, y desde el punto de vista militar, tales cuerpos especiales encontraron su razón de ser en las características de su tiempo y también en la organización interna de un ejército romano cada vez más

79. Véase Speidel 2020 (2021), 421-431.

80. Aur. Víct., Caes. 39, 44; medida muy alabada por el autor, dentro de la imagen ya positiva que ofrece en su obra del reinado de Diocleciano.

81. Santos Yanguas 1977, 127-139; también Sinnigen 1959, 238-254, y Sinnigen 1964, 78-105; Arias Bonet 1957-58, 197-220; González Salinero 2008, 191-207.

82. McCunn 2019, 13-15. 
especializado ${ }^{83}$. Los legionarios elegidos como frumentarii tuvieron que mostrar cualidades innatas que a ojos de sus oficiales les hicieron recomendables para desgajarse de la legión y realizar cometidos distintos. ¿Cómo actuó tal designación en los propios frumentarii? Indudablemente de modo positivo; se creó, como se ha visto, un espíritu de cuerpo, que se desarrolló posiblemente desde el principio.

No debe olvidarse que los frumentarii pertenecían a un servicio de inteligencia que era casi enteramente militar. Para todos los ejércitos, el cometido más importante de la inteligencia es descubrir la ubicación y número del enemigo. Esto incluía la supervisión detallada de rutas terrestres, comunicación fluvial y el control de puntos de referencia como hórreos, torres, puentes o granjas fortificadas ${ }^{84}$. Controlando tales cuestiones, y teniendo acceso a cartas, correspondencia y documentos, como mensajeros, resulta lógico que la información atesorada los convirtiese casi de inmediato en candidatos perfectos para vigilar y controlar el resto de cuestiones de interés para el poder. Recordemos que estas tropas estaban siempre en movimiento, de ahí su devoción a Júpiter, deidad a la que pedían un buen retorno de sus numerosos viajes y misiones, y protección en los caminos ${ }^{85}$. Las habilidades secundarias que potenciaban sus dotes para el secretismo o la ocultación hicieron el resto, por lo que también pudieron ser empleados satisfactoriamente en misiones de todo tipo relacionadas con la infiltración, mientras que el gran abanico proporcionado por su adiestramiento militar especial les preparaba cuando las tareas les movían a deshacerse de un rival, usando la fuerza siempre que resultase necesario. A nuestro juicio, su empleo y desarrollo a finales del siglo II y principios del siglo III les muestran en posesión de una serie de habilidades distintivas que los pusieron en valor como elementos de gran utilidad en las confrontaciones bélicas, sobre todo civiles, gracias a un fuerte carácter de especialistas militares. Terminaremos este trabajo con la reflexión de G. Cupcea, que consideramos acertada: "Good for anything, the frumentarii are as important as any other military for the proper function of the Roman states ${ }^{86}$.

83. Speidel 2009, 283-304.

84. Sheldon 2006, 199-249; Russell 2013, 474-493.

85. Faure 2003, 413-414; véase también CIL VI 428. Se hace allí referencia a un templo de Iuppiter Redux en el Castra Peregrinorum.

86. Cupcea 2009, 313. 
6. Bibliografía

Appelbaum, Alan. "Another Look at the Assassination of Pertinax and the Accession of Julianus». CPh 102.2 (2007): 198-207.

Arias Bonet, Juan Antonio. "Los agentes in rebus: contribución al estudio de la policía en el Bajo Imperio romano». AHDE 27-28 (1957-1958): 197-220.

Austin, Norman James Edmonstone y Nikolas Boris Rankov. Exploratio. Military and Political Intelligence in the Roman World from the Second Punic War to the Battle of Adrianopole. London \& New York: Routledge, 1995.

Baillie Reynolds, Paul Kenneth. "The troops quartered in the castra peregrinorum». JRS 13 (1923): 168-189.

Bennett, Julian. Trajan Optimus Princeps. A Life and Times. London: Routledge, 2005.

Bérard, François. (2015): L'armée romaine à Lyon (Bibliothèque des Écoles françaises d'Athènes et de Rome, 370). Roma: École française de Rome.

Bingham, Sandra. The Praetorian Guard: A History of Rome's Elite Special Forces. London: Bloomsbury Publishing, 2013.

Birley, Arthur Richard. "The Coups d'Etat of year 192». BJ 169 (1969): 247280.

Birley, Arthur Richard. Septimio Severo: el emperador africano. Traducción de José Luis Gil Aristu. Madrid: Gredos, 1971, 2012.

Birley, Eric Barff. "Military Intelligence in the Historia Augusta». En Bonner Historia-Augusta-Colloquium 1964/1965, editado por Andreas Alföldi, 35-42. Bonn: Rudolf Habelt Verlag, 1966.

Breeze, David John. "Pay Grades and Ranks below the Centurionate». JRS 61 (1971): 130-135.

Brunt, Peter Astbury. "Pay and Superannuation in the Roman Army". PBSR 18 (1950): 50-71.

Busch, Alexandra Wilhelmine. "Militia in Urbe». The military presence in Rome». En The Impact of the Roman Army (200 BC - AD 476): Economic, Social, Political, Religious and Cultural Aspects. Proceedings of the Sixth Workshop of the International Network "Impact of Empire (Roman Empire, 200 B.C. - A.D. 476)", Capri, Italy, March 29-April 2, 2005. Impact of Empire, Volume 6, editado por Lukas de Blois y Elio Lo Cascio, 315-341. Leiden, Brill, 2007.

Clauss, Manfred. Untersuchungen zu den principales des römischen Heeres von Augustus bis Diokletian. Cornicularii, speculatores, frumentarii. Bochum: Ruhr-Universität, Diss. 1973. 
Coarelli, Filippo y Luisanna Usai. Guida archeologica di Roma. Roma: Arnoldo Mondadori Editore, 1984.

Cowan, Ross y Angus McBride. Imperial Roman Legionary, AD 161-284. Oxford: Osprey Publishing, 2003.

Crowdy, Terry. The enemy within. Oxford: Osprey Publishing, 2006.

Cupcea, George. "The missions of the soldiers in the limes provinces. Frumentarii in Dacia”. En Near and Beyond. The Roman Frontiers, editado por Ovidiu Țentea y Ioan Carol Opriş, 305-314. Bucharest: National History Museum of Romania, 2009.

Dvornik, Francis. Origins of Intelligence Services: The Ancient Near East, Persia, Greece, Rome, Byzantium, the Arab Muslim Empires, the Mongol Empire, China, Muscovy. New Brunswick: Rutgers University Press, 1974.

Eck, Werner. Die Verwaltung des römischen Reiches in der hohen Kaiserzeit. Basel: F. Reinhardt Verlag, 1995.

Faure, Patrice. "Les centurions frumentaires et le commandement des castra peregrina». MEFRA 115(1) (2003): 377-427.

Fiebiger, Heinrich Otto. "Frumentarii». En Paulys Realencyclopädie der classischen Altertumswissenschaft. Stuttgart: Metzler Verlag, 1912.

Forni, Giovanni. Il Reclutamento Delle Legioni Da Augusto a Diocleziano. Milano: Fratelli Bocca, 1953.

Fraipont, Mathieu. Le numerus frumentariorum romain: un outil de la gouvernance impériale des IIe et IIIe siècles. Faculté de philosophie, arts et lettres, Université catholique de Louvain, 2019. Prom: Van Haeperen.

Fuhrmann, Christopher J. Policing the Roman Empire: Soldiers, Administration, and Public Order. New York and Oxford: Oxford University Press, 2012.

González Salinero, Raúl. «Investigadores de la corrupción, corruptos: la degradación moral de los agentes in rebus». En La corrupción en el mundo romano. Actas del IV Coloquio de la Asociación Interdisciplinar de Estudios Romanos, editado por Gonzalo Bravo Castañeda y Raúl González Salinero, 191-207. Madrid: Signifer Libros, 2008.

Hirt, Alfred Michael. Imperial Mines and Quarries in the Roman World: Organizational Aspects 27 BC-AD 235. New York and Oxford: Oxford University Press, 2010.

Kagan, Kimberly. "Spies Like Us: Treason and Identity in the Late Roman Empire». En Romans, Barbarians, and the Transformation of the Roman World. Cultural Interaction and the Creation of Identity in Late Antiquity, editado por Ralph Whitney Mathisen y Danuta Shanzer, 161173. Farnham: Asghate, 2011. 
Kemezis, Adam M. "Cassius Dio and Senatorial Memory of Civil War in the 190s». En Cassius Dio: The Impact of Violence, War, and Civil War, editado por Carsten Hjort Lange y Andrew G. Scott, 257-286. Leiden: Brill, 2020.

Kolb, Anne. Transport und Nachrichtentransfer im römischen Reich. Klio / Beihefte. Neue Folge, 2. Berlin: Akademie Verlag, 2000. DOI: https:// doi.org/10.1524/9783050048246

Krenkel, Werner. "Frumentarii». En Lexicon der Alten Welt. Zurich, Munich: Artemis Verlag, 1990.

Lee, Alan Douglas. Information and Frontiers. Roman Foreign Relations in Late Antiquity. Cambridge: Cambridge University Press, 1993, 2006.

Liberati, Anna Maria y Enrico Silverio. Servizi segreti in Roma antica. Informazioni e sicurezza dagli initia Urbis all'impero universale. Roma: "L'Erma» di Bretschneider, 2010.

McCunn, Stuart. "What's in a name? The evolving role of the frumentarii». CQ 69.1 (2019): 340-354.

Mann, John Cecil. "The Organization of the Frumentarii». ZPE 74 (1988): 149-150.

Martines, Lauro. April Blood: Florence and the Plot against the Medici. Oxford: Oxford University Press, 2003.

Martini, Giovanni. «I milites frumentarii». AIV 139 (1980-1981): 143-151.

Matijevic, Ivan. "Qui cucurrit frumentarius annos XI". Tusculum: časopis za solinske teme 7.1 (2014): 64-74.

Menéndez Argüín, Adolfo Raúl. "II Parthica: legio apud Romam». Habis 34 (2003): 313-321.

Menéndez Argüín, Adolfo Raúl. "Administración de la logística militar romana durante el Principado (ss. I-III d.C.)». Florllib. 17 (2006): 153167.

Millar, Fergus. A Study of Cassius Dio. Oxford: The Clarendon Press, 1964.

Nelis-Clément, Jocelyne. Les Beneficiarii. Militaires et administrateurs au service de l'Empire (Ier s. a. C. - VIe s. p. C.). Bordeaux: Ausonius Éditions, 2000.

Nutt, David C. "Silvanus and the emperor Constantius II». Antichthon 7 (1973): 80-89.

Oliver, James Henry. «M. Aquilius Felix». AJPh 67.4 (1946): 311-319.

Padilla Monge, Aurelio. La provincia romana de la Bética (253-422). Écija: Editorial Gráficas Sol, 1989, 2019.

Palao Vicente, Juan José (2016): «La organización de la inteligencia militar y de los "servicios secretos" en el ejército romano". En Congreso Internacional de la Cátedra Extraordinaria Complutense de Historia Militar, "la Organización de los ejércitos", Madrid, 2015, coordinado 
por Lola Sánchez Lázaro, 123-158. Madrid: Cátedra Extraordinaria Complutense de Historia Militar. Gobierno de España, Ministerio de Defensa.

Panciera, Silvio. "Castra peregrina. Vecchi e nuovi documenti epigrafici». En Epigrafi, Epigrafia, Epigrafisti. Scritti vari editi e inediti (19562005) con note complementari e indici, Silvio Panciera, 1471-1482. Roma: Quasar, 2006.

Panciera, Silvio. "Signis legionum. Insegne, immagini imperiali e centuriones frumentarii a peregrinis». En Epigrafi, Epigrafia, Epigrafisti. Scritti vari editi e inediti (1956-2005) con note complementari e indici, 14531464. Roma: Quasar, 2006.

Paribeni, Roberto. "Dei milites frumentarii e dell approvigionamento della corte imperiale». $\operatorname{MDAI}(R) 20$ (1905): 310-320.

Paschoud, François. «Frumentarii, agentes in rebus, magistriani, curiosi, veredarii: problèmes de terminologie». En Bonner Historia-AugustaColloquium 1979/1981. editado por Géza Alföldy, Johannes Straub et al., 215-244. Bonn: R. Habelt Verlag, 1983.

Penwill, John Leonard. «Evolution of an Assassin: The Letters of Chion of Heraclea». Ramus 39(1) (2010): 24-52. DOI: https://doi.org/10.1017/ S0048671X00000527

Perea Yébenes, Sabino. Collegia Militaria. Asociaciones militares en el Imperio romano (Monografías y Estudios de Antigüedad Griega y Romana). Madrid: Signifer, 1999, 2013.

Perea Yébenes, Sabino. "Tronos ensangrentados (Ca. 251-350). Quiebra del sacramentum militar y traición: de la securitas Augusti al crimen maiestatis". En Tradimento e traditori nella Tarda Antichità, editado por Luca Montecchio, 1-34. Perugia: Edizioni Graphé, 2017.

Perea Yébenes, Sabino. «La prodigiosa vista y el fino oído de los Mauri y su utilización ocasional en la inteligencia militar y en la exploratio. A propósito de Julio Africano, Cesti VII, 16». CaSteR 5, (2020): on line. DOI: https://doi.org/10.13125/caster/4132

Petraccia, Maria Federica. In rebus agere. Il mestiere di spia nell'antica Roma. Bologna: Pàtron, 2012.

Rantala, Jussi. "Dio the Dissident: The Portrait of Severus in the Roman History». En Cassius Dio: Greek Intellectual and Roman Politician, editado por Jesper Majbom Madsen y Carsten Hjort Lange, 159-177. Leiden: Brill, 2016.

Reuter, Marcus. "Die frumentarii, neugeschaffene 'Geheimpolizei' Traians". En Traian in Germanien-Traian im Reich. Bericht des dritten Saalburgkolloquiums, editado por Egon Schallmayer, 77-81. Bad Homburg, v.d.H.: Saalburgmuseum, 1999. 
Rankov, Nikolas Boris. "M. Oclatinius Adventus in Britain». Britannia 18 (1987): 243-49. DOI: https://doi.org/10.2307/526449

Rankov, Nikolas Boris. "Frumentarii, the Castra Peregrina and the provincial officia». ZPE 80 (1990): 176-182.

Rankov, Nikolas Boris. "Les frumentarii et la circulation de l'information entre les empereurs romains et les provinces». En La circulation de l'information dans les États antiques, Actes de La circulation de l'information dans les structures de pouvoir antiques (Pessac 2002) (Études, n. ${ }^{\circ}$ 14), editado por Laurent Capdetrey y Jocelyne Nelis-Clément, 129140. Bordeaux: Ausonius, 2006.

Rankov, Nikolas Boris. "The origins of the frumentarii». En XII Congressus Internationalis Epigraphiae Graecae et Latinae: Provinciae Imperii Romani Inscriptionibus Descriptae, editado por Giulia Baratta, Alejandra Guzmán Almagro y Marc Mayer i Olivé, 1169-1172. Barcelona: Institut d'Estudis Catalans, 2007.

Rickman, Geoffrey. Roman Granaries and Store Buildings. Cambridge: Cambridge University Press, 1971.

Rodríguez González, Julio. La dinastía de los Severos: el comienzo del declive del Imperio Romano. Madrid: Almena Ediciones, 2010.

Russell, Frank. "Finding the Enemy: Military Intelligence». En The Oxford Handbook of Warfare in the Classical World, editado por Brian Campbell y Lawrence A. Tritle, 474-493. New York and Oxford: Oxford University Press, 2013).

Santos Yanguas, Narciso. "El servicio policial secreto romano en el Bajo Imperio según Ammiano Marcelino». MHA 1 (1977): 127-139.

Sheldon, Rose Mary. Intelligence Activities in Ancient Rome. London and New York: Routledge, 2005.

Sheldon, Rose Mary. Intelligence Activities in Ancient Rome: Trust in the Gods but Verify. Abingdon \& New York: Routledge, 2006.

Sheldon, Rose Mary. Espionage in the Ancient World: An Annotated Bibliography of Books and Articles in Western Languages. Jefferson (NC): McFarland \& Company Inc. Publishers, 2008.

Sinnigen, William Gurnee. "Two Branches of the Late Roman Secret Service». AJPh 80(3) (1959): 238-254.

Sinnigen, William Gurnee. «The Roman Secret Service». CJ 57(2) (1961): 65-72.

Sinnigen, William Gurnee. "The Origins of the Frumentari». MAAR 27 (1962): 211-224. DOI: https://doi.org/10.2307/4238654

Sinnigen, William Gurnee. "Chiefs of Staff of the Secret Service». ByzZ 57 (1964): 78-105. 
Southern, Patricia. «The numeri of the Roman imperial army». Britannia 20 (1989): 81-140.

Speidel, Michael Alexander. «Roman Army Pay Scales». JRS 82 (1992): 87-106.

Speidel, Michael Alexander. «Specialisation and Promotion in the Roman Imperial Army". En Administration, Prosopography and Appointment Policies in the Roman Empire. Proceedings of the First Workshop of the International Network Impact of Empire (Roman Empire, 27 B.C. - A.D. 406), Leiden, June 28-July 1, 2000. Impact of Empire, Volume I, editado por Lukas de Blois, 50-61. Leiden: Brill, 2001. DOI: https://doi. org/10.1163/9789004401617_007

Speidel, Michael Alexander. «Specialisation and Promotion in the Roman Imperial Army». En Heer und Herrschaft im Römischen Reich der Hohen Kaiserzeit, editado por Michael Alexander Speidel, 283-304. Stuttgart: Franz Steiner Verlag, 2009.

Speidel, Michael Alexander. "Protectors and Assassins. Caracalla's guards on the day he died". En Corps du chef et gardes du corps dans l'armée romaine. Actes du septième Congrès de Lyon (25-27 octobre 2018), (Collection études et recherches sur l'Occident Romain: CEROR), editado por Catherine Wolff y Patrice Faure, 421-431. Lyon: Diffusion Librairie De Boccard 11, rue Médicis, Paris, 2020, 2021.

Speidel, Michael Paul. "Exploratores. Mobile Elite Units of Roman Germany». Epigraphische Studien 13 (1983): 63-78.

Speidel, Michael Paul. Die Denkmäler der Kaiserreiter. Equites Singulares Augusti. Beihefte der Bonner Jahrbücher, Band 50. Eine Veröffentlichung des Landschaftsverbandes. Köln: Rheinland-Verlag, 1993.

Speidel, Michael Paul. Riding for Caesar: The Roman Emperors' Horse Guard. Cambridge, Mass.: Harvard University Press, 1994, 2002.

Syme, Ronald. Ammianus and the Historia Augusta. New York and Toronto: Oxford University Press, 1968.

Syme, Ronald. «Marius Maximus Once Again». En Bonner Historia-AugustaColloquium 1970, editado por András Alföldi, Elisabeth AlföldiRosenbaum, Géza Alföldy et al., 287-302. Bonn: R. Habelt Verlag, 1972. Syme, Ronald. Historia Augusta Papers. New York and Oxford: The Clarendon Press, 1983.

Vaglieri, Dante. «Frumentarii». En Dizionario Epigrafico di Antichita Romane. Roma: Tipografia della R. Accademia dei Lincei, 1922.

Van Sickle, Clifton Edwin. "The Legal Status of Clodius Albinus in the Years 193-96». CPh 23.2 (1928): 123-127.

Von Domaszewski, Alfred. Die Rangordnung des römischen Heeres (Ed. B. Dobson). Köln-Graz: Böhlau, 1967. 